\title{
REPRESENTATIONS OF RECIPROCALS OF LUCAS SEQUENCES
}

\section{H. R. HASHIM AND SZ. TENGELY}

Received 08 February, 2018

Abstract. In 1953 Stancliff noted an interesting property of the Fibonacci number $F_{11}=89$. One has that

$$
\frac{1}{89}=\frac{F_{0}}{10}+\frac{F_{1}}{10^{2}}+\frac{F_{2}}{10^{3}}+\frac{F_{3}}{10^{4}}+\frac{F_{4}}{10^{5}}+\frac{F_{5}}{10^{6}}+\cdots .
$$

De Weger determined a complete list of similar identities in case of the Fibonacci sequence, the solutions are as follows

$$
\begin{array}{cc}
\frac{1}{F_{1}}=\frac{1}{F_{2}}=\frac{1}{1}=\sum_{k=1}^{\infty} \frac{F_{k-1}}{2^{k}}, & \frac{1}{F_{5}}=\frac{1}{5}=\sum_{k=1}^{\infty} \frac{F_{k-1}}{3^{k}}, \\
\frac{1}{F_{10}}=\frac{1}{55}=\sum_{k=1}^{\infty} \frac{F_{k-1}}{8^{k}}, & \frac{1}{F_{11}}=\frac{1}{89}=\sum_{k=1}^{\infty} \frac{F_{k-1}}{10^{k}} .
\end{array}
$$

In this article we study similar problems in case of general Lucas sequences $U_{n}(P, Q)$. We deal with equations of the form

$$
\frac{1}{U_{n}\left(P_{2}, Q_{2}\right)}=\sum_{k=1}^{\infty} \frac{U_{k-1}\left(P_{1}, Q_{1}\right)}{x^{k}},
$$

for certain pairs $\left(P_{1}, Q_{1}\right) \neq\left(P_{2}, Q_{2}\right)$. We also consider equations of the form

$$
\sum_{k=1}^{\infty} \frac{U_{k-1}(P, Q)}{x^{k}}=\sum_{k=1}^{\infty} \frac{R_{k-1}}{y^{k}},
$$

where $R_{n}$ is a ternary linear recurrence sequence. The proofs are based on results related to Thue equations and elliptic curves.

2010 Mathematics Subject Classification: 11D25; 11B39

Keywords: Lucas sequences, Diophantine equations, elliptic curves

This work was partially supported by the European Union and the European Social Fund through project EFOP-3.6.1-16-2016-00022 (Sz.T.). The research was supported in part by grant K115479 and K128088 (Sz.T.) of the Hungarian National Foundation for Scientific Research. The work of Hayder H. R. was supported by the Stipendium Hungaricum Scholarship. 


\section{INTRODUCTION}

Let $P$ and $Q$ be non-zero relatively prime integers. The Lucas sequence $\left\{U_{n}(P, Q)\right\}$ is defined by

$$
U_{0}=0, U_{1}=1 \text { and } U_{n}=P U_{n-1}-Q U_{n-2}, \text { if } n \geq 2 .
$$

The associated Lucas sequence $\left\{V_{n}(P, Q)\right\}$ is defined by

$$
V_{0}=2, V_{1}=P \text { and } V_{n}=P U_{n-1}-Q U_{n-2} \text {, if } n \geq 2 \text {. }
$$

Terms of Lucas sequences and associated Lucas sequences satisfy the identity

$$
V_{n}^{2}-D U_{n}^{2}=4 Q^{n}
$$

where $D=P^{2}-4 Q$. In 1953, Stancliff [12] noted an interesting property of the Fibonacci sequence $U_{n}(1,-1)=F_{n}$. One has that

$$
\frac{1}{F_{11}}=\frac{1}{89}=0.0112358 \ldots=\sum_{k=0}^{\infty} \frac{F_{k}}{10^{k+1}} .
$$

In 1980, Winans [17] studied the related sums

$$
\sum_{k=0}^{\infty} \frac{F_{\alpha k}}{10^{k+1}}
$$

for certain values of $\alpha$. In 1981 Hudson and Winans [7] characterized all decimal fractions that can be approximated by sums of the type

$$
\frac{1}{F_{\alpha}} \sum_{k=1}^{n} \frac{F_{\alpha k}}{10^{l(k+1)}}, \quad \alpha, l \geq 1 .
$$

Long [10] obtained a general identity for binary recurrence sequences from which one obtains e.g.

$$
\frac{1}{109}=\sum_{k=0}^{\infty} \frac{F_{k}}{(-10)^{k+1}}, \quad \frac{1}{10099}=\sum_{k=0}^{\infty} \frac{F_{k}}{(-100)^{k+1}} .
$$

In case of the equation

$$
\frac{1}{U_{n}(P, Q)}=\sum_{k=1}^{\infty} \frac{U_{k-1}(P, Q)}{x^{k}},
$$

De Weger [4] determined all $x \geq 2$ in case of $(P, Q)=(1,-1)$. The solutions are as follows

$$
\frac{1}{F_{1}}=\frac{1}{F_{2}}=\frac{1}{1}=\sum_{k=1}^{\infty} \frac{F_{k-1}}{2^{k}}, \quad \frac{1}{F_{5}}=\frac{1}{5}=\sum_{k=1}^{\infty} \frac{F_{k-1}}{3^{k}}
$$




$$
\frac{1}{F_{10}}=\frac{1}{55}=\sum_{k=1}^{\infty} \frac{F_{k-1}}{8^{k}}, \quad \frac{1}{F_{11}}=\frac{1}{89}=\sum_{k=1}^{\infty} \frac{F_{k-1}}{10^{k}} .
$$

In 2014 Tengely [15] extended the above result and obtained e.g.

$$
\frac{1}{U_{10}}=\frac{1}{416020}=\sum_{k=0}^{\infty} \frac{U_{k}}{647^{k+1}},
$$

where $U_{0}=0, U_{1}=1$ and $U_{n}=4 U_{n-1}+U_{n-2}, n \geq 2$. Recently Ohtsuka and Nakamura [11] proved that

$$
\left\lfloor\left(\sum_{k=n}^{\infty} \frac{1}{F_{k}}\right)^{-1}\right\rfloor= \begin{cases}F_{n-2} & \text { if } n \geq 2 \text { is even, } \\ F_{n-2}-1 & \text { if } n \geq 1 \text { is odd, }\end{cases}
$$

where $\lfloor\cdot\rfloor$ denotes the floor function. This result has been investigated by several other mathematicians see e.g. [6,9].

\section{Auxiliary RESUlts}

In the proofs we will use the following two results of Köhler [8].

Lemma 1. Let $A, B, a_{0}, a_{1}$ be arbitrary complex numbers. Define the sequence $\left\{a_{n}\right\}$ by the recursion $a_{n+1}=A a_{n}+B a_{n-1}$. Then the formula

$$
\sum_{k=0}^{\infty} \frac{a_{k}}{x^{k+1}}=\frac{a_{0} x-A a_{0}+a_{1}}{x^{2}-A x-B}
$$

holds for all complex $x$ such that $|x|$ is larger than the absolute values of the zeros of $x^{2}-A x-B$.

Lemma 2. Let arbitrary complex numbers $A_{0}, A_{1}, \ldots, A_{m}, a_{0}, a_{1}, \ldots, a_{m}$ be given. Define the sequence $\left(a_{n}\right)_{n}$ by the recursion

$$
a_{n+1}=A_{0} a_{n}+A_{1} a_{n-1}+\cdots+A_{m} a_{n-m}
$$

Then for all complex $z$ such that $|z|$ is larger than the absolute values of all zeros of $q(z)=z^{m+1}-A_{0} z^{m}-A_{1} z^{m-1}-\cdots-A_{m}$, the formula

$$
\sum_{k=1}^{\infty} \frac{a_{k-1}}{z^{k}}=\frac{p(z)}{q(z)}
$$

holds with $p(z)=a_{0} z^{m}+b_{1} z^{m-1}+\cdots+b_{m}$, where $b_{k}=a_{k}-\sum_{i=0}^{k-1} A_{i} a_{k-1-i}$ for $1 \leq k \leq m$. 


\section{MAIN RESULTS}

In this paper we extend the results of [15], we consider the equation

$$
\frac{1}{U_{n}\left(P_{2}, Q_{2}\right)}=\sum_{k=1}^{\infty} \frac{U_{k-1}\left(P_{1}, Q_{1}\right)}{x^{k}},
$$

for certain pairs $\left(P_{1}, Q_{1}\right) \neq\left(P_{2}, Q_{2}\right)$. We consider non-degenerate sequences with $1 \leq P \leq 3$ and $Q= \pm 1$. Define the set $S$ as follows

$$
\begin{aligned}
S= & \left\{u_{1}(n)=U_{n}(1,-1), u_{2}(n)=U_{n}(1,1), u_{3}(n)=U_{n}(2,-1), u_{4}(n)=U_{n}(3,-1),\right. \\
& \left.u_{5}(n)=U_{n}(3,1)\right\} .
\end{aligned}
$$

Theorem 1. The equation

$$
\frac{1}{u_{j}(n)}=\sum_{k=1}^{\infty} \frac{u_{i}(k-1)}{x^{k}},
$$

has the following solutions with $1 \leq i, j \leq 5, i \neq j$

$$
\begin{aligned}
& (i, j, n, x) \in\{(1,2,\{1,2\}, 2),(1,3,1,2),(1,3,3,3),(1,3,5,6),(1,4,1,2), \\
& (1,4,5,11),(1,4,7,35),(1,5,1,2),(1,5,5,8),(2,1,4,2),(2,1,7,4), \\
& (2,1,8,5),(2,5,2,2),(2,5,4,5),(3,1,3,3),(3,1,9,7),(4,1,4,4), \\
& (4,1,14,21),(4,5,2,4),(4,5,7,21),(5,1,\{1,2\}, 3),(5,1,5,4), \\
& (5,1,10,9),(5,1,11,11),(5,2,\{1,2\}, 3),(5,3,1,3),(5,3,3,4), \\
& (5,3,5,7),(5,4,1,3),(5,4,5,12),(5,4,7,36)\} .
\end{aligned}
$$

We also deal with equations of the form

$$
\sum_{k=1}^{\infty} \frac{u_{j}(k-1)}{x^{k}}=\sum_{k=1}^{\infty} \frac{R_{k-1}}{y^{k}},
$$

where $R_{n}$ is a ternary linear recurrence sequence. We provide results in case of the Tribonacci sequence defined by $T_{0}=T_{1}=0, T_{2}=1$ and $T_{n+3}=T_{n+2}+T_{n+1}+$ $T_{n}, n \geq 0$ and Berstel's sequence, that is given by $B_{0}=B_{1}=0, B_{2}=1$ and $B_{n+3}=$ $2 B_{n+2}-4 B_{n+1}+4 B_{n}, n \geq 0$.

Theorem 2. The complete list of solutions of equation (3.3) with $u_{n} \in S, R_{n} \in$ $\left\{B_{n}, T_{n}\right\}$ and positive integers $x, y$ satisfying conditions of Lemma 1 and 2 is as follows 


\begin{tabular}{|c|c|c||c|c|c|}
\hline$u_{n}$ & $R_{n}$ & $(x, y)$ & $u_{n}$ & $R_{n}$ & $(x, y)$ \\
\hline \hline$u_{1}$ & $B_{n}$ & $\{(25,9)\}$ & $u_{1}$ & $T_{n}$ & $\{(2,2)\}$ \\
\hline$u_{2}$ & $B_{n}$ & $\{(10,5)\}$ & $u_{2}$ & $T_{n}$ & $\{(7,4),(309,46)\}$ \\
\hline$u_{3}$ & $B_{n}$ & \{\} & $u_{3}$ & $T_{n}$ & $\left\{\left(t\left(t^{2}-2\right)+1, t^{2}-1\right): t \geq 2, t \in \mathbb{N}\right\}$ \\
\hline$u_{4}$ & $B_{n}$ & $\{(6,3),(18,7)\}$ & $u_{4}$ & $T_{n}$ & \{\} \\
\hline$u_{5}$ & $B_{n}$ & $\{(26,9)\}$ & $u_{5}$ & $T_{n}$ & \{\} \\
\hline
\end{tabular}

\section{PROOFS OF THE THEOREMS}

Proof of Theorem 1. Consider equation (3.1), by Lemma 1 we obtain that

$$
\sum_{k=1}^{\infty} \frac{U_{k-1}\left(P_{1}, Q_{1}\right)}{x^{k}}=\frac{1}{x^{2}-P_{1} x+Q_{1}} .
$$

Hence we have that $U_{n}\left(P_{2}, Q_{2}\right)=x^{2}-P_{1} x+Q_{1}$. Combining the latter equation with (1.1) we get $V_{n}\left(P_{2}, Q_{2}\right)^{2}=\left(P_{2}^{2}-4 Q_{2}\right)\left(x^{2}-P_{1} x+Q_{1}\right)^{2}+4 Q_{2}^{n}$. The socalled two-cover descent by Bruin and Stoll [3] can be used to prove that a given hyperelliptic curve has no rational points. It is implemented in Magma [2], the procedure is called TwoCoverDescent. If it fails and we do not find any rational points on the curve, then we apply the argument by Alekseyev and Tengely [1], that reduces the problem to Thue equations. If we have a rational point on the curve, then using a method by Tzanakis [16] the integral points can be determined. This algorithm is implemented in Magma as IntegralquarticPoints. In this way we collect the possible values of $x$.

\begin{tabular}{|c|c||c|c||c|c|}
\hline$\left(P_{1}, Q_{1}, P_{2}, Q_{2}\right)$ & $x$ & $\left(P_{1}, Q_{1}, P_{2}, Q_{2}\right)$ & $x$ & $\left(P_{1}, Q_{1}, P_{2}, Q_{2}\right)$ & $x$ \\
\hline \hline$(1,-1,1,1)$ & 2 & $(1,1,1,-1)$ & $2,4,5$ & $(2,-1,1,-1)$ & 3,7 \\
\hline$(1,-1,2,-1)$ & $2,3,6$ & $(1,1,2,-1)$ & - & $(2,-1,1,1)$ & - \\
\hline$(1,-1,3,-1)$ & $2,11,35$ & $(1,1,3,-1)$ & 2 & $(2,-1,3,-1)$ & - \\
\hline$(1,-1,3,1)$ & 2,8 & $(1,1,3,1)$ & 2,5 & $(2,-1,3,1)$ & - \\
\hline
\end{tabular}

\begin{tabular}{|c|c||c|c|}
\hline$\left(P_{1}, Q_{1}, P_{2}, Q_{2}\right)$ & $x$ & $\left(P_{1}, Q_{1}, P_{2}, Q_{2}\right)$ & $x$ \\
\hline$(3,-1,1,-1)$ & 4,21 & $(3,1,1,-1)$ & $3,4,9,11$ \\
\hline$(3,-1,1,1)$ & - & $(3,1,1,1)$ & 3 \\
\hline$(3,-1,2,-1)$ & - & $(3,1,2,-1)$ & $3,4,7$ \\
\hline$(3,-1,3,1)$ & 4,21 & $(3,1,3,-1)$ & $3,12,36$ \\
\hline
\end{tabular}

It remains to compute the set of possible values of $n$. We provide details of the computation in case of $\left(P_{1}, Q_{1}, P_{2}, Q_{2}\right)=(3,-1,1,-1)$, following these steps all other equations can be handled. In case of $\left(P_{1}, Q_{1}, P_{2}, Q_{2}\right)=(3,-1,1,-1)$ we have that $x \in\{4,21\}$. If $x=4$, then we define a matrix $T$ as follows

$$
T=\left(\begin{array}{cc}
3 / 4 & 1 / 4 \\
1 / 4 & 0
\end{array}\right)
$$


We have that

$$
\frac{1}{4}\left(T^{0}+T^{1}+T^{2}+\cdots+T^{N-1}\right)\left(\begin{array}{l}
1 \\
0
\end{array}\right)=\left(\begin{array}{c}
* \\
\sum_{k=1}^{N} \frac{U_{k-1}(3,-1)}{4^{k}}
\end{array}\right) .
$$

It follows that

$$
\begin{aligned}
& \sum_{k=1}^{N} \frac{U_{k-1}(3,-1)}{4^{k}}= \\
& -\frac{2^{-3 N-1}}{39}\left((\sqrt{13}+3)^{N}(5 \sqrt{13}+13)+(13-5 \sqrt{13})(-\sqrt{13}+3)^{N}-13 \cdot 2^{3 N+1}\right),
\end{aligned}
$$

hence we have that

$$
\lim _{N \rightarrow \infty} \sum_{k=1}^{N} \frac{U_{k-1}(3,-1)}{4^{k}}=\frac{1}{3}=\frac{1}{U_{4}(1,-1)}
$$

In this case we obtain that $n=4$. If $x=21$, then

$$
T=\left(\begin{array}{cc}
3 / 21 & 1 / 21 \\
1 / 21 & 0
\end{array}\right)
$$

In a similar way than in case of $x=4$ we get that

$$
\begin{aligned}
& \sum_{k=1}^{N} \frac{U_{k-1}(3,-1)}{21^{k}}= \\
& \quad \frac{\left(7^{N} 3^{N} 2^{N+1}-(\sqrt{13}+3)^{N}(3 \sqrt{13}+1)+(3 \sqrt{13}-1)(-\sqrt{13}+3)^{N}\right) 2^{-N-1}}{377 \cdot 7^{N} 3^{N}},
\end{aligned}
$$

therefore

$$
\lim _{N \rightarrow \infty} \sum_{k=1}^{N} \frac{U_{k-1}(3,-1)}{21^{k}}=\frac{1}{377}=\frac{1}{U_{14}(1,-1)} .
$$

The only solution in this case is given by $n=14$.

Proof of Theorem 2. We provide a general argument that works for other sequences as well. Let $a_{0}=0, a_{1}=1$ and $a_{n+1}=A a_{n}+B a_{n-1}$. Let $b_{0}=b_{1}=0, b_{2}=1$ and $b_{n+1}=C b_{n}+D b_{n-1}+E b_{n-2}$. Equation (3.3) yields that

$$
Y^{2}=X^{3}-4 C X^{2}-16 D X+16 A^{2}+64 B-64 E,
$$

where $Y=8 x-4 A$ and $X=4 y$. If the cubic polynomial in $X$ is square-free, then we have an elliptic equation and integral points can be determined using the socalled elliptic logarithm method developed by Stroeker and Tzanakis [14] and independently by Gebel, Pethő and Zimmer [5]. There exists a number of software implementations for determining integral points on elliptic curves based on this technique, here we used SageMath [13]. Let us consider the case with $u_{2}(n), T_{n}$. We 
obtain the elliptic curve $Y^{2}=X^{3}-4 X^{2}-16 X-112$. Using the SageMath function integral_points () we get

$$
[(8: 4: 1),(16: 52: 1),(29: 143: 1),(184: 2468: 1)] \text {. }
$$

From these points we have that $(x, y) \in\{(7,4),(309,46)\}$. As a second example consider the case with $u_{4}, B_{n}$. The elliptic curve is given by $Y^{2}=X^{3}-8 X^{2}+64 X-48$. The list of integral points is

$$
[(1: 3: 1),(4: 12: 1),(12: 36: 1),(28: 132: 1)] .
$$

Thus we get that $(x, y) \in\{(6,3),(18,7)\}$. Finally let us deal with the special case with $u_{3}, T_{n}$. The cubic polynomial is not square-free, it is $(X+4)(X-4)^{2}$. Therefore we have that $X+4=4 y+4=u^{2}$. Hence $y=t^{2}-1$ for some integer $t \geq 2$. It follows that $x=t\left(t^{2}-2\right)+1$. So we obtain infinitely many identities of the form

$$
\sum_{k=1}^{\infty} \frac{u_{4}(k-1)}{\left(t\left(t^{2}-2\right)+1\right)^{k}}=\sum_{k=1}^{\infty} \frac{T_{k-1}}{\left(t^{2}-1\right)^{k}} .
$$

\section{ACKNOWLEDGEMENT}

The authors express their gratitude to the referee for careful reading of the manuscript and many valuable suggestions, which improve the quality of this paper.

\section{REFERENCES}

[1] M. A. Alekseyev and S. Tengely, "On integral points on biquadratic curves and near-multiples of squares in lucas sequences,” J. Integer Seq., vol. 17, no. 6, pp. Article 14.6.6, 15, 2014.

[2] W. Bosma, J. Cannon, and C. Playoust, "The Magma algebra system. I. The user language," J. Symbolic Comput., vol. 24, no. 3-4, pp. 235-265, 1997, computational algebra and number theory (London, 1993), doi: 10.1006/jsco.1996.0125. [Online]. Available: https://doi.org/10.1006/jsco.1996.0125

[3] N. Bruin and M. Stoll, "Two-cover descent on hyperelliptic curves," Math. Comp., vol. 78, no. 268, pp. 2347-2370, 2009, doi: 10.1090/S0025-5718-09-02255-8. [Online]. Available: http://dx.doi.org/10.1090/S0025-5718-09-02255-8

[4] B. M. M. de Weger, "A curious property of the eleventh Fibonacci number," Rocky Mountain J. Math., vol. 25, no. 3, pp. 977-994, 1995, doi: 10.1216/rmjm/1181072199. [Online]. Available: http://dx.doi.org/10.1216/rmjm/1181072199

[5] J. Gebel, A. Pethő, and H. G. Zimmer, "Computing integral points on elliptic curves." Acta Arith., vol. 68, no. 2, pp. 171-192, 1994, doi: 10.4064/aa-68-2-171-192. [Online]. Available: https://doi.org/10.4064/aa-68-2-171-192

[6] S. H. Holliday and T. Komatsu, "On the sum of reciprocal generalized Fibonacci numbers." Integers, vol. 11, no. 4, pp. 441-455, a11, 2011, doi: 10.1515/INTEG.2011.031.

[7] R. H. Hudson and C. F. Winans, "A complete characterization of the decimal fractions that can be represented as $\sum 10^{-k(i+1)} F_{\alpha i}$, where $F_{\alpha i}$ is the $\alpha i$ th Fibonacci number," Fibonacci Quart., vol. 19 , no. 5 , pp. 414-421, 1981.

[8] G. Köhler, "Generating functions of Fibonacci-like sequences and decimal expansions of some fractions," Fibonacci Quart., vol. 23, no. 1, pp. 29-35, 1985. 
[9] K. Kuhapatanakul, "On the sums of reciprocal generalized Fibonacci numbers." J. Integer Seq., vol. 16, no. 7, pp. article 13.7.1, 8, 2013.

[10] C. T. Long, "The decimal expansion of 1/89 and related results," Fibonacci Quart., vol. 19, no. 1, pp. 53-55, 1981.

[11] H. Ohtsuka and S. Nakamura, "On the sum of reciprocal Fibonacci numbers." Fibonacci Q., vol. 46-47, no. 2, pp. 153-159, 2009.

[12] F. Stancliff, "A curious property of $a_{i i}$," Scripta Math., vol. 19, p. 126, 1953.

[13] W. Stein et al., Sage Mathematics Software (Version 8.1), The Sage Development Team, 2018, http://www. sagemath.org.

[14] R. J. Stroeker and N. Tzanakis, "Solving elliptic diophantine equations by estimating linear forms in elliptic logarithms." Acta Arith., vol. 67, no. 2, pp. 177-196, 1994, doi: 10.4064/aa-67-2-177-196. [Online]. Available: https://doi.org/10.4064/aa-67-2-177-196

[15] S. Tengely, "On the Lucas sequence equation $\frac{1}{U_{n}}=\sum_{k=1}^{\infty} \frac{U_{k-1}}{x^{k}}$." Period. Math. Hung., vol. 71, no. 2, pp. 236-242, 2015, doi: 10.1007/s10998-015-0101-4.

[16] N. Tzanakis, "Solving elliptic Diophantine equations by estimating linear forms in elliptic logarithms. The case of quartic equations," Acta Arith., vol. 75, no. 2, pp. 165-190, 1996, doi: 10.4064/aa-75-2-165-190. [Online]. Available: https://doi.org/10.4064/aa-75-2-165-190

[17] C. F. Winans, "The Fibonacci series in the decimal equivalents of fractions," in A collection of manuscripts related to the Fibonacci sequence. Fibonacci Assoc., Santa Clara, Calif., 1980, pp. $78-81$.

\section{Authors' addresses}

\section{H. R. Hashim}

Mathematical Institute, University of Debrecen, P.O.Box 12, 4010 Debrecen, Hungary

E-mail address: hayderr.almuswi@uokufa.edu.iq

\section{Sz. Tengely}

Mathematical Institute, University of Debrecen, P.O.Box 12, 4010 Debrecen, Hungary

E-mail address: tengelyescience. unideb.hu 